\title{
THE METHOD OF BILINEAR PROGRAMMING FOR NONCONVEX QUADRATIC PROGRAMMING
}

1. Introduction. The subject of this paper is the solution method of the nonconvex quadratic programming problem (problem NQP) with a nonnegative definite quadratic form. The set of its feasible solutions is a convex polyhedral set, not necessarily bounded. The problem may be stated as follows :

Problem NQP. Maximize

$$
G(x)=h^{T} x+x^{T} Q x
$$

subject to

$$
\boldsymbol{x} \in X=\left\{\boldsymbol{x} \in R^{n} \mid A \boldsymbol{x}=\boldsymbol{c}, \boldsymbol{x} \geqslant \boldsymbol{O}\right\},
$$

where $Q$ is a symmetric nonnegative definite matrix of order $n$, rank $A$ $=k \leqslant n, \boldsymbol{h} \in R^{n}$ and $c \in R^{k}$.

Problem NQP belongs to a class of mathematical programming problems having many local maxima in the vertices of the set $X$. Hence, its optimal solution is in some vertex of $X$. This results from the convexity of the function $G(x)$.

Problem NQP may be solved by the algorithm of bilinear programming (given in [3]) modified to the form corresponding to problem BLP1 stated in Section 2 of this paper. The possibility of solving the problem NQP by an algorithm of bilinear programming has been also noticed in papers [1], [6] and [8]. However, due to some important properties of problem NQP, presented also in Section 2, we state such necessary and sufficient conditions for the existence of an optimal solution the verification of which is reduced to the solution of problem LP (linear programming) only; this result is in contrast to problem BLP (bilinear programming). Thus, an algorithm for solving the problem NQP, presented in Section 3, is simplified drastically in relation to the method of bilinear programming.

In most methods dealing with problem NQP one usually assumes that the set of feasible solutions is bounded (compare with [6] and [8]). In papers [2] and [9], only the existence of an optimal solution is assumed 
but both of the procedures require finding $\max \{g(x) \mid x \in X\}$, where $g(x)$ is the function defined by (2.11) in this paper. Unfortunately (as our Example 1 shows), the existence of such a maximum is not even a consequence of the existence of an optimal solution of problem NQP. Thus the lack of some additional assumptions (such as, for example, boundedness of the set $X$ ) makes the methods proposed in [2] and [9] incorrect.

On the other hand, in [2] it is additionally assumed that there exist values $s_{j}$ (defined by formula (2.1) of this paper). But this fact, in the case of problem NQP, is a simple consequence of the boundedness of the function $G(x)$ (see Corollary 4). Let us notice that in the case of problem BLP, which (according to the author's claim) may be solved by the same method, the existence of $s_{j}$ is only a sufficient condition for the existence of an optimal solution of problem BLP (see Theorem 6 in [3]).

In paper [1] the general problem NQP is considered but the method proposed for solving the problem BLP fails to be proper. More exact remarks concerning paper [1] are given in Section 1 of [3].

2. General properties of problem NQP. We start with discussing the relations between problems NQP and BLP. Let us consider the following Problem BLP1. Maximize

$$
F(x, y)=\frac{1}{2} h^{T} x+\frac{1}{2} h^{T} y+y^{T} Q x
$$

subject to $(\boldsymbol{x}, \boldsymbol{y}) \in X \times X$, where $\boldsymbol{h}, Q, X$ are defined as in problem NQP.

This problem is a particular case of problem BLP, discussed in [3], where $A=B, \boldsymbol{a}=\boldsymbol{b}=\frac{1}{2} \boldsymbol{h} \in R^{n}, \boldsymbol{c}=\boldsymbol{d} \in R^{k}$ and, consequently, $X=Y$.

Problem NQP is equivalent to the following

Problem NQP1. Maximize $(*)$ subject to $(\boldsymbol{x}, \boldsymbol{y}) \in \boldsymbol{X} \times \boldsymbol{X}$ and $\boldsymbol{x}=\boldsymbol{y}$.

The equivalence of problems NQP and NQP1 is established in the following obvious remark:

Remark 1. The point $x^{0}$ is an optimal solution of problem NQP if and only if the point $\left(x^{0}, x^{0}\right)$ is an optimal solution of problem NQP1.

Clearly, problem NQP1 is not a problem BLP because the condition $\boldsymbol{x}=\boldsymbol{y}$ appears. However, the following theorem allows us to use the bilinear programming algorithm for the solution of problem NQP.

THEOREM 1. If $\left(x^{0}, y^{0}\right)$ is an optimal solution of problem BLP1, then also the points $\left(\boldsymbol{y}^{0}, \boldsymbol{x}^{0}\right),\left(\boldsymbol{x}^{0}, \boldsymbol{x}^{0}\right),\left(\boldsymbol{y}^{0}, \boldsymbol{y}^{0}\right) \in X \times X$ are optimal solutions of the problem.

Proof. By the symmetry of the function $F(x, y)$ we have $F\left(x^{0}, y^{0}\right)$ $=F\left(y^{0}, x^{0}\right)$, which implies the optimality of $\left(y^{0}, x_{0}\right)$. 
Let $z^{0}=F\left(x^{0}, y^{0}\right)=F\left(y^{0}, x^{0}\right)$. Then

$$
F\left(x^{0}, x^{0}\right)+F\left(y^{0}, y^{0}\right)-F\left(x^{0}, y^{0}\right)-F\left(y^{0}, x^{0}\right)=\left(y^{0}-x^{0}\right)^{\mathrm{T}} Q\left(y^{0}-x^{0}\right) \geqslant 0,
$$

since $Q$ is nonnegative definite. Hence

$$
F\left(x^{0}, x^{0}\right)+F\left(y^{0}, y^{0}\right) \geqslant 2 z^{0} .
$$

But, on the other hand, it is known that

$$
z^{0}=\max \{\boldsymbol{F}(\boldsymbol{x}, \boldsymbol{y}) \mid(\boldsymbol{x}, \boldsymbol{y}) \in X \times X\}
$$

thus

$$
F\left(x^{0}, x^{0}\right) \leqslant z^{0} \quad \text { and } \quad F\left(y^{0}, y^{0}\right) \leqslant z^{0}
$$

From the last three inequalities it follows that $F\left(x^{0}, x^{0}\right)=F\left(y^{0}, y^{0}\right)$ $=z^{0}$. Thus the points $\left(x^{0}, x^{0}\right)$ and $\left(y^{0}, y^{0}\right)$ are optimal solutions of problem BLP1. The proof is completed.

From the proof of Theorem 1 we obtain

Corollary 1. Let $Q$ be positive definite. If the point $\left(x^{0}, y^{0}\right)$ is an optimal solution of problem $B L P 1$, then $x^{0}=y^{0}$.

Remark 1 and Theorem 1 show that all properties of problem BLP may be transferred to problem NQP.

Let $X_{\mathrm{p}}$ denote (as in [3]) a convex hull of all vertices of the set $X$ and let $X_{c}$ be a convex polyhedral cone of the form

$$
X_{\mathrm{c}}=\left\{\boldsymbol{x} \in R^{n} \mid A \boldsymbol{x}=\boldsymbol{0}, \boldsymbol{x} \geqslant \boldsymbol{0}\right\} .
$$

A special version of Theorem 1 from [3] is the following

THEOREM 2. The function $G(x)$ is bounded from above on the set of feasible solutions of problem NQP if and only if the following conditions hold:

(i) $\boldsymbol{x}^{\mathrm{T}} \boldsymbol{Q} \boldsymbol{x}=\mathbf{0}$ for $\boldsymbol{x} \in \boldsymbol{X}_{\mathrm{o}}$;

(ii) $\frac{1}{2} h^{\mathrm{T}} \boldsymbol{x}+\boldsymbol{y}^{\mathrm{T}} Q \boldsymbol{x} \leqslant 0$ for $(\boldsymbol{x}, \boldsymbol{y}) \in X_{\mathrm{c}} \times X_{\mathrm{p}}$.

Proof. Let $x \in X_{\mathrm{c}}$ and $y \in X_{\mathrm{p}}$. The thesis follows from the fact that $y+\lambda x \in X$ for any $\lambda \geqslant 0$ and

$$
G(y+\lambda x)=G(y)+\lambda\left(h^{T} x+2 y^{T} Q x\right)+\lambda^{2} x^{T} Q x .
$$

Let us notice that the equality in (i) follows from the nonnegative definiteness of $Q$.

From Theorem 2 and the definition of problem NQP we obtain

Corollary 2. Let $Q$ be positive definite. Then the function $G(x)$ is bounded from above on the set $X$ if and only if the set $X$ is bounded.

Proof. The sufficiency follows from the Weierstrass theorem. To show the necessity suppose that $X$ is unbounded. Then $X_{c} \neq\{0\}$ and for $\bar{x} \in X_{c}-\{0\}$ we have $\bar{x}^{\mathrm{T}} Q \overline{\boldsymbol{x}}>0$ since $Q$ is positive definite which, by (i) of Theorem 2, implies that the function $G(x)$ is unbounded from above on the set $X$. This contradiction completes the proof. 
Theorem 3. The function $G(x)$ is bounded from above on the set $X$ if and only if the linear form $\boldsymbol{h}^{\mathrm{T}} \boldsymbol{x}$ and the quadratic form $\boldsymbol{x}^{\mathrm{T}} Q \boldsymbol{Q}$ are also bounded from above on $X$.

Proof. The sufficiency follows from the inequality

$$
\max \{G(x) \mid x \in X\} \leqslant \max \left\{\boldsymbol{h}^{\mathbf{T}} \boldsymbol{x} \mid \boldsymbol{x} \in X\right\}+\max \left\{\boldsymbol{x}^{\mathbf{T}} \boldsymbol{Q} \boldsymbol{x} \mid \boldsymbol{x} \in X\right\} .
$$

Let us suppose now that the quadratic form $x^{\mathrm{T}} Q \boldsymbol{x}$ is unbounded from above on the set $X$. Then there exists a point $\bar{x} \in X_{\mathrm{c}}$ such that $\overline{\boldsymbol{x}}^{\mathrm{T}} Q \overline{\boldsymbol{x}}>0$. Hence the function $G(x)$ is unbounded from above on the set $X$ according to condition (i) of Theorem 2.

Now, let us notice that for any $x \in R^{n}$ the inequality

$$
\boldsymbol{h}^{\mathrm{T}} \boldsymbol{x} \leqslant \boldsymbol{h}^{\mathrm{T}} \boldsymbol{x}+\boldsymbol{x}^{\mathrm{T}} \boldsymbol{Q} \boldsymbol{x}=\boldsymbol{G}(\boldsymbol{x})
$$

holds, since $Q$ is nonnegative definite. Therefore, if $\boldsymbol{h}^{\mathbf{T}} \boldsymbol{x}$ is unbounded from above on the set $X$, then $G(x)$ is also unbounded from above on $X$. This contradiction completes the proof of the necessity.

In order to state further properties of problem NQP we prove some properties of a nonnegative definite matrix $Q=\left[q_{j i}\right]$.

LEMMA 1. If $Q$ is a nonnegative definite matrix and if there exists $q_{j j}=0$ for some $j \in\{1, \ldots, n\}$, then the $j$-th column and the $j$-th row of the matrix $Q$ are zero vectors.

Proof. Let $q_{j j}=0$ and $q_{j i} \neq 0$ for some $i \neq j$. Taking the point $\overline{\boldsymbol{x}} \in R^{n}$ with coordinates

$$
\bar{x}_{l}= \begin{cases}M & \text { for } l=j \\ 1 & \text { for } l=i, \\ 0 & \text { for } l \in\{1, \ldots, n\}-\{i, j\}\end{cases}
$$

we have $\overline{\boldsymbol{x}}^{\mathrm{T}} Q \overline{\boldsymbol{x}}=q_{i i}+2 q_{j i} M$.

It is easy to choose such an $M$ that $\bar{x}^{\mathrm{T}} Q \bar{x}<0$ for given $q_{i i}$ and $q_{j i} \neq 0$, which contradicts the assumption that $Q$ is nonnegative definite. This completes the proof.

Since all elements $q_{j j}$ of the main diagonal of a nonnegative definite matrix $Q$ are nonnegative, we obtain the following corollary:

CoRollary 3. If $Q$ is a nonnegative definite matrix and if $q_{j i} \neq 0$ for some $j \neq i$, then $q_{j j}>0$ and $q_{i i}>0$.

Now, let us introduce a vector $s \in R^{n}$ with coordinates defined by the formula

$$
s_{j}=\max \left\{\boldsymbol{q}_{j} \boldsymbol{x} \mid \boldsymbol{x} \in X\right\} \quad \text { for } j \in\{1, \ldots, n\},
$$

where $\boldsymbol{q}_{j}$ denotes the $j$-th row (equal to the $j$-th column) of the matrix $Q$.

THEOREM 4. The nonnegative definite quadratic form $\boldsymbol{x}^{\mathrm{T}} Q \boldsymbol{x}$ is bounded from above on the set $X$ if and only if $s_{j}<+\infty$ for all $j \in\{1, \ldots, n\}$. 
Proof. We can assume $q_{j j}>0$, since otherwise we would have $\boldsymbol{s}_{j}=0$ (according to Lemma 1). Applying the first step of the Lagrange method for transforming the quadratic form $\boldsymbol{x}^{\mathrm{T}} \boldsymbol{Q} \boldsymbol{x}$ into its diagonal form, we obtain (see [4])

$$
\boldsymbol{x}^{\mathrm{T}} Q \boldsymbol{x}=\frac{1}{q_{j j}}\left(\sum_{i=1}^{n} q_{j i} x_{i}\right)^{2}+f\left(x_{1}, \ldots, x_{j-1}, x_{j+1}, \ldots, x_{n}\right),
$$

where $f$ is a nonnegative definite quadratic form of $n-1$ variables. Hence, if

$$
\boldsymbol{s}_{j}=\max \left\{\sum_{i=1}^{n} q_{j i} x_{i} \mid x \in X\right\}=+\infty
$$

then the quadratic form $x^{\mathrm{T}} Q x$ would be unbounded from above on the set $X$. This proves the necessity.

To prove the sufficiency, let us notice the following property of problem LP:

$$
\boldsymbol{s}_{j}<+\infty \quad \text { implies } \quad \bar{s}_{j}=\max \left\{\boldsymbol{q}_{j} \boldsymbol{x} \mid \boldsymbol{x} \in \boldsymbol{X}_{\mathrm{c}}\right\}=0 \text {. }
$$

Hence $0 \leqslant x^{\mathrm{T}} Q \boldsymbol{x} \leqslant \overline{\boldsymbol{s}}^{\mathrm{T}} \boldsymbol{x}=\mathbf{0}$ for any $x \in X_{\mathrm{c}}$ and $\boldsymbol{y}^{\mathrm{T}} \boldsymbol{Q} \boldsymbol{x} \leqslant \overline{\boldsymbol{s}}^{\mathrm{T}} \boldsymbol{y}=\mathbf{0}$ for any $(x, y) \in X_{c} \times X_{p}$, which implies, by Theorem 2 while $h=0$, that the quadratic form $\boldsymbol{x}^{\mathrm{T}} Q \boldsymbol{x}$ is bounded from above on the set $X$. This completes the proof.

The following corollary is an immediate consequence of Theorems 3 and 4:

Corollary 4. The function $G(x)$ is bounded from above on the set of feasible solutions of problem NQP if and only if

$$
\max \left\{\boldsymbol{h}^{\mathrm{T}} \boldsymbol{x} \mid \boldsymbol{x} \in X\right\}<+\infty \quad \text { and } \quad s_{j}<+\infty \text { for all } j \in\{1, \ldots, n\} .
$$

In our further description we use the notation introduced in [3].

Let $\alpha=\left\{j_{1}, \ldots, j_{k}\right\} \subset\{1, \ldots, n\}$ and $\xi=\{1, \ldots, n\}-\alpha$ denote the sets of indices of basic and nonbasic variables, respectively, which correspond to the basis $A_{a}=\left[A_{\left\{j_{1}\right\}}, \ldots, A_{\left\{j_{k}\right\}}\right]$ constructed from linearly independent columns of the matrix $A$ spanning $R^{k}$. Similarly, $x_{a}$ and $x_{\xi}$ denote the subvectors of the vector $x$ consisting of coordinates with indices belonging to the sets $\alpha$ and $\xi$, respectively, and $A_{\xi}$ denotes the submatrix of $A$ formed from the columns of $A$ with indices belonging to the set $\xi$.

Let $\boldsymbol{x}^{a}$ denote the basic feasible solution corresponding to the basis $A_{\alpha}$ (i.e. $\left.x_{\xi}^{a}=0\right)$. Then the function $\Delta(x)=G(x)-G\left(x^{a}\right)$ is of the form

$$
\Delta(x)=2 \boldsymbol{p}_{\xi}^{\mathrm{T}} x_{\xi}+x_{\xi}^{\mathrm{T}} V_{\xi \xi} \boldsymbol{x}_{\xi},
$$


where

$$
\begin{gathered}
\boldsymbol{p}_{\xi}=\frac{1}{2} \boldsymbol{h}_{\xi}+Q_{\xi}^{\mathrm{T}} x^{a}+E_{\xi}^{\mathrm{T}}\left(\frac{1}{2} h_{a}+Q_{a}^{\mathrm{T}} x^{a}\right) \\
V_{\xi \xi}=E_{\xi}^{\mathrm{T}}\left(Q_{a \alpha} E_{\xi}-Q_{\alpha \xi}\right)-Q_{\xi \alpha} E_{\xi}+Q_{\xi \xi}
\end{gathered}
$$

and $E_{\xi}=A_{\alpha}^{-1} A_{\xi}$ (see [3]).

Remark 2. The matrix $V_{\xi \xi}$ is nonnegative definite since $Q$ is nonnegative definite. Moreover, if the quadratic form $x^{\mathbf{T}} \boldsymbol{Q} x$ is bounded from above on the set $X$, then the quadratic form $x_{\xi}^{\mathrm{T}} \nabla_{\xi \xi} x_{\xi}$ is also bounded from above on $X$.

Remark 3. Analogously to Remark 3 from [3], a point $x^{\alpha} \in X$ is an optimal solution of problem NQP if and only if $\max \{\Delta(x) \mid x \in X\}$ $=0$.

Due to Corollary 4, the boundedness of the function $G(x)$ can be examined before an optimal solution is sought. Therefore, in the remainder of this section we assume that the function $G(x)$ is bounded from above on the set $X$.

Let $\xi^{0}=\left\{j \in \xi \mid E_{\{j\}} \leqslant 0\right\}$.

Proposition 1. If the function $G(x)$ is bounded from above on the set $X$, then $V_{\{j\}\{j\}}=0$ and $p_{j} \leqslant 0$ for all $j \in \xi^{0}$.

Proof. Suppose that $V_{\{j\}\{j\}}>0$ or $p_{j}>0$ for some $j \in \xi^{0}$ and consider the points $x(\lambda)$ defined by $x(\lambda)=\lambda \bar{x}$, where $\bar{x}_{a}=-E_{\{j\}}, \bar{x}_{j}=1$, $\overline{\boldsymbol{x}}_{\xi-\{j\}}=\boldsymbol{0}, \lambda \geqslant \mathbf{0}$.

From formula (2.2) for $x^{a}+x(\lambda) \in X$ we obtain

$$
\Delta\left(x^{a}+x(\lambda)\right)=p_{j} \lambda+\lambda^{2} \nabla_{\{j\} j\}}>0 \quad \text { whenever } \lambda>0 .
$$

Hence

$$
\lim _{\lambda \rightarrow+\infty} \Delta\left(\boldsymbol{x}^{a}+\boldsymbol{x}(\lambda)\right)=+\infty
$$

This contradicts the assumption that $G(x)$ is bounded from above on the set $X$.

Let us denote (as in [3]) by $\alpha^{\prime}$ the set of indices of basic variables such that $a^{\prime}-a=\{j\}$, where $j \in \xi-\xi^{0}$, and introduce the following values:

$$
v_{j}=\min \left\{x_{l}^{\alpha}\left|E_{\{l\}\{j\}}\right| E_{\{l\}\{j\}}>0, l \in \alpha\right\} .
$$

Then the basic feasible solution $x^{a^{\prime}}$ adjacent to the basic feasible solution $x^{\alpha}$ is defined by

$$
x_{j}^{a^{\prime}}=v_{j}, \quad x_{\xi-\{j\}}^{a^{\prime}}=0, \quad x_{\alpha}^{a^{\prime}}=x_{\alpha}^{\alpha}-v_{j} E_{\{j\}} .
$$


Proposition 2. If $p_{j}>0$ for some $j \in \xi-\xi^{0}$, then $G\left(x^{a^{\prime}}\right) \geqslant G\left(x^{a}\right)$. Moreover, if $\boldsymbol{x}^{\alpha}$ is nondegenerate, then $G\left(\boldsymbol{x}^{\alpha^{\prime}}\right)>G\left(\boldsymbol{x}^{\alpha}\right)$.

Proof. Let us notice that $G\left(x^{\alpha}\right)=F\left(x^{\alpha}, x^{\alpha}\right)$ and $G\left(x^{\alpha^{\prime}}\right)=F\left(x^{a^{\prime}}, x^{\alpha^{\prime}}\right)$. From Proposition 2 in [3] we get $F\left(x^{\alpha}, x^{a}\right) \leqslant F\left(x^{\alpha^{\prime}}, x^{\alpha}\right)$ and from Proposition 3 in [3] we obtain $F\left(x^{\alpha^{\prime}}, x^{\alpha}\right) \leqslant F\left(x^{\alpha^{\prime}}, x^{a^{\prime}}\right)$. Thus the proposition is proved.

Proposition 3. If $2 p_{j}+v_{j} V_{\{j\}\{j\}}>0$ holds for some $j \in \xi-\xi^{0}$, then $G\left(x^{a^{\prime}}\right)>G\left(x^{a}\right)$ whenever $v_{j}>0$.

Proof. The proposition follows from the simple observation that $\Delta\left(x^{a^{\prime}}\right)=2 p_{j} v_{j}+v_{j}^{2} V_{\{j\}\{j\}}$ and from Remark 2 in [3].

THEOREM 5. If the point $x^{\alpha} \in X$ is a basic optimal solution of problem $N Q P$, then the following conditions hold:

$$
\begin{gathered}
\boldsymbol{p}_{\xi} \leqslant \mathbf{0}, \\
2 p_{j}+v_{j} V_{\{j\} j\}} \leqslant 0 \quad \text { for all } j \in \xi-\xi^{0}, \\
\nabla_{\{j\} j\}}=0 \quad \text { for all } j \in \xi^{0} .
\end{gathered}
$$

The theorem results directly from Propositions 1, 2 and 3.

COROLLARY 5. If for the basic feasible solution $\boldsymbol{x}^{a}$ condition (2.7) holds and $\nabla_{\{j\} j\}}=0$ for all $j \in \xi$, then $x^{\alpha}$ is an optimal solution of problem NQP.

Proof. Due to Lemma 1 the equalities $V_{\{j\} ;\}}=0$ for all $j \in \xi$ imply $V_{\xi \xi}=[0]$. Thus, if $\boldsymbol{p}_{\xi} \leqslant \boldsymbol{0}$, then $\Delta(\boldsymbol{x}) \leqslant 0$ for any $\boldsymbol{x} \in X$, which by Remark 3 completes the proof.

In order to state sufficient conditions for optimality we introduce the vector $z_{\xi}$ whose coordinates are defined by

$$
z_{j}=\max \left\{V_{\{j\}} x_{\xi} \mid x \in X\right\} \quad \text { for } j \in \xi,
$$

where $V_{\{j\}}$ denotes the $j$-th row of the matrix $V_{\xi \xi}$.

Let us also define a function $g$ by

$$
g(\boldsymbol{x})=\left(2 \boldsymbol{p}_{\xi}+\boldsymbol{z}_{\xi}\right)^{\mathrm{T}} \boldsymbol{x}_{\xi} .
$$

It should be stressed that, in contrast to problem BLP, the existence of values $z_{j}$ defined by (2.10) is a consequence of the boundedness of $G(x)$ on the set $X$. This fact follows from Corollary 4 and Remark 2 . It can be easily proved that $z_{j} \geqslant 0$ for all $j \in \xi$ and $\Delta(x) \leqslant g(x)$ for $x \in X$. However, it should be emphasized that the function $g(x)$ defined by (2.11) may be unbounded from above on the set $X$ even in the case where an optimal solution of problem NQP exists (i.e. if the function $\Delta(x)$ and also $G(x)$ are bounded from above on $X$ ). This is clarified by the following example: 
Example 1. Let us consider problem (1.1)-(1.2) in which $h=0 \in R^{4}$, $c=\left[\begin{array}{ll}1 & 1\end{array}\right]^{\mathrm{T}}$, and

$$
Q=\left[\begin{array}{rrrr}
1 & -1 & 0 & 0 \\
-1 & 1 & 0 & 0 \\
0 & 0 & 0 & 0 \\
0 & 0 & 0 & 0
\end{array}\right], \quad A=\left[\begin{array}{rrrr}
1 & -1 & 1 & 0 \\
-1 & 1 & 0 & 1
\end{array}\right]
$$

Let us notice that the set of feasible solutions of the problem can be expressed as follows:

$$
X=\left\{x \in R^{4} \mid x_{1}-x_{2}+x_{3}=1,-x_{1}+x_{2}+x_{4}=1, x \geqslant 0\right\} .
$$

Taking then $a=\{3,4\}$ and $\xi=\{1,2\}$ we have

$$
\begin{aligned}
& x^{a}=\left[\begin{array}{l}
0 \\
0 \\
1 \\
1
\end{array}\right], \quad Q_{\xi}=\left[\begin{array}{rr}
1 & -1 \\
-1 & 1 \\
0 & 0 \\
0 & 0
\end{array}\right], \quad Q_{a}=\left[\begin{array}{ll}
0 & 0 \\
0 & 0 \\
0 & 0 \\
0 & 0
\end{array}\right], \quad E_{\xi}=\left[\begin{array}{ll}
1 & 0 \\
0 & 1
\end{array}\right], \\
& Q_{a a}=\left[\begin{array}{ll}
0 & 0 \\
0 & 0
\end{array}\right], \quad Q_{\xi \xi}=\left[\begin{array}{rr}
1 & -1 \\
-1 & 1
\end{array}\right] \quad \text { and } \quad Q_{a \xi}=Q_{\xi a}=\left[\begin{array}{ll}
0 & 0 \\
0 & 0
\end{array}\right]
\end{aligned}
$$

Thus from (2.3) we get the vector $p_{\xi}=\left[\begin{array}{ll}0 & 0\end{array}\right]^{\mathrm{T}}$ and from (2.4) we obtain the matrix

$$
V_{\xi \xi}=\left[\begin{array}{rr}
1 & -1 \\
-1 & 1
\end{array}\right]
$$

It is easy to verify that $x_{\xi}^{\mathrm{T}} \nabla_{\xi \xi} x_{\xi}=\left(x_{1}-x_{2}\right)^{2} \geqslant 0$ for each $x \in X$. Then the matrix $V_{\xi \xi}$ and also (due to Remark 2) the matrix $Q$ are nonnegative definite.

Obviously, $\max \left\{\boldsymbol{p}_{\xi}^{\mathbf{T}} \boldsymbol{x}_{\xi} \mid \boldsymbol{x} \in \boldsymbol{X}\right\}=\mathbf{0}$.

Applying the simplex method we obtain (according to formula (2.10)) the following values:

$$
\begin{aligned}
& z_{1}=\max \left\{x_{1}-x_{2} \mid x \in X\right\}=1<+\infty \\
& z_{2}=\max \left\{-x_{1}+x_{2} \mid x \in X\right\}=1<+\infty
\end{aligned}
$$

Thus, due to Corollary 4 and Remark 2, the functions $\Delta(x)$ and also $G(x)$ are bounded from above on the set $X$. But, according to formula (2.11), $g(x)=x_{1}+x_{2}$ and $\max \left\{x_{1}+x_{2} \mid x \in X\right\}=+\infty$ although an optimal solution of the considered problem exists.

Let $X^{*}$ denote the sequence of all vertices of $X$ ordered in such a manner that $g\left(x^{i}\right) \geqslant g\left(x^{j}\right)$ whenever $i<j$ for $x^{i}, x^{j} \in X^{*}$, obtained by the algorithm from [7] (or [4] whenever $\max \{\boldsymbol{g}(\boldsymbol{x}) \mid \boldsymbol{x} \in \boldsymbol{X}\}=+\infty$ ). 
Theorem 6. The basic feasible solution $x^{a}$ is optimal if at least one of the following conditions holds:

$$
\begin{aligned}
& 2 \boldsymbol{p}_{\xi}+z_{\xi} \leqslant 0 ; \\
& \text { the sequence } \boldsymbol{x}^{1}, \ldots, \boldsymbol{x}^{r} \in X^{*} \text { satisfies } g\left(\boldsymbol{x}^{r}\right) \leqslant 0 \text { and } \\
& \qquad \max \left\{\Delta(\boldsymbol{x}) \mid \boldsymbol{x} \in\left\{\boldsymbol{x}^{1}, \ldots, \boldsymbol{x}^{r-1}\right\}\right\} \leqslant 0 .
\end{aligned}
$$

Proof. From (2.10), (2.11) and from $\Delta(x) \leqslant g(x)$ we obtain $\Delta(x)$ $\leqslant g(x) \leqslant 0$ for any $x \in X$, which proves the sufficiency of (2.12) (compare with Remark 3 ).

Since $\Delta(x) \leqslant g(x)$ for any $x \in X$ and $g\left(x^{r}\right) \leqslant 0$, we have $\Delta(x) \leqslant 0$ for $x \in X^{*}-\left\{x^{1}, \ldots, x^{r-1}\right\}$. From (2.13) we get also $\Delta(x) \leqslant 0$ for $x \in\left\{x^{1}, \ldots, x^{r-1}\right\}$. Therefore, $\Delta(x) \leqslant 0$ for any $x \in X$, which, by Remark 3, proves the sufficiency of (2.13). This completes the proof.

3. An algorithm for solving the problem NQP. The results obtained in Section 2 enable us to propose an algorithm for finding an optimal solution of problem NQP.

The algorithm presented in this paper consists of two distinct parts. In Steps 1 and 2 the boundedness of the function $G(x)$ is examined according to Corollary 4.

In the second part (Steps 3-9) an optimal solution is sought knowing that the function $G(x)$ is bounded from above on the set $X$. It should be stressed that according to Proposition 1 further computations may be restricted to the set $\xi-\xi^{0}$.

In Steps 4-6 the conditions of Theorem $\tilde{5}$ and Corollary 5 are used while the conditions of Theorem 6 are verified in Steps 7-9.

In Step 7 it may be necessary to use the method from [4] for maximizing a linear form over a convex hull of vertices of the set $X$ while in Step 9 we must apply the method from [7] for ranking vertices of a convex polyhedral set in a sequence for which the values of a linear form do not increase (or the more general method from [4]).

All other steps utilize standard linear programming procedures.

It should be stressed that any method working under the assumption of the existence of an optimal solution of problem NQP may be used instead of the second part of our algorithm.

\section{The algorithm}

Step 1. Find $h_{0}=\max \left\{\boldsymbol{h}^{\mathrm{T}} \boldsymbol{x} \mid \boldsymbol{x} \in X\right\}$. If $h_{0}<+\infty$, go to Step 2. Otherwise, the function $G(x)$ is unbounded from above on the set $X$.

Step 2. Find $s_{j}$ for $j \in\left\{j \in\{1, \ldots, n\} \mid q_{j j}>0\right\}$ defined by (2.1). If $s_{j}<+\infty$ for all $j$, go to Step 3. Otherwise, the function $G(x)$ is unbounded from above on $X$. 
Step 3. Find (1) a basic feasible solution $x^{\alpha} \in X$.

Step 4. Compute the vector $p_{\xi}$ according to (2.3). If (2.7) holds, go to Step 5. Otherwise, pick $j \in \xi-\xi^{0}$ such that $p_{j}=\max \left\{p_{l}>0 \mid l \in \xi-\xi^{0}\right\}$, compute $v_{j}$ according to (2.5), replace $x^{a}$ by $x^{a^{\prime}}$ obtained from (2.6) and repeat Step 4.

Step 5. Compute the matrix $V_{\xi \xi}$ according to (2.4). If $V_{\{j\} j\}}=0$ for all $j \in \xi-\xi^{0}$, then $x^{\alpha}$ is an optimal solution of problem NQP. Otherwise, select the set $\xi^{1}=\left\{j \in \xi-\xi^{0} \mid V_{\{j\}\{j\}}>0\right\}$.

Step 6. Compute the values $v_{j}$ for $j \in \xi^{1}$ according to (2.5). If conditions (2.8) hold, go to Step 7. Otherwise, pick $j \in \xi^{1}$ such that $2 p_{j}+v_{j} V_{\{j\} j\}}$ $>0$, replace $x^{\alpha}$ by $x^{a^{\prime}}$ obtained from (2.6) and return to Step 4.

Step 7. Find the values $z_{j}$ for $j \in \xi^{1}$ according to (2.10). If conditions (2.12) hold for all $j \in \xi^{1}$, then $x^{\alpha}$ is an optimal solution of problem NQP. Otherwise, find the vertex $x^{1} \in X^{*}$ maximizing the function $g(x)$ (defined by (2.11)) on the set $X_{\mathrm{p}}$.

Step 8. If $g\left(x^{1}\right)>0$, go to Step 9. Otherwise, $x^{a}$ is an optimal solution of problem NQP.

Step 9. Compute $\Delta\left(x^{1}\right)$. If $\Delta\left(x^{1}\right) \leqslant 0$, find the vertex $x^{2} \in X^{*}$ which follows directly $x^{1}$, replace $x^{1}$ by $x^{2}$ and return to Step 8. Otherwise, replace $x^{\alpha}$ by $x^{1}$ and return to Step 4 .

Remark 4 . Since the number of vertices of the set $X$ is finite and the algorithm does not generate new vertices, it terminates in a finite number of iterations yielding an optimal solution of problem NQP.

\section{References}

[1] M. Altman, Bilinear programming, Bull. Acad. Polon. Sci., Sér. Sci. Math. Astronom. Phys., 16 (1968), p. 741-746.

[2] A. V. Cabot and R. L. Francis, Solving certain nonconvex quadratic minimization problems by ranking extreme points, Operations Res. 18 (1970), p. 82-86.

[3] I. Czochralska, Bilinear programming, this fascicle p. 495-514.

[4] - and W. Grabowski, Maximization of a linear form over a convex hull of vertices of a convex polyhedral set, Zastos. Mat. 18 (1983) (to appear).

[5] G. Hadley, Linear algebra, Addison-Wesley, Reading 1961.

[6] H. Konno, Maximization of a convex quadratic function under linear constraints, Math. Programming 11 (1976), p. 117-127.

[7] K. G. Murty, Solving the fixed charge problems by ranking the extreme points, Operations Res. 16 (1968), p. 268-279.

${ }^{1}$ ) Select $x^{a}$ from solutions obtained in Steps 1 and 2 with respect to the greatest value of the objective function $G(x)$. 
[8] E. N. Sokirjanskaja (Е. Н. Сокирянская), Некоторые алгоритмы билинейного программирования, Кибернетика 4 (1974), р. 106-112.

[9] H. A. Tah a, Concave minimization over a convex polyhedron, Naval Res. Logist. Quart. 20 (1973), p. 533-548.

DEPARTMENT OF MATHEMATICS

CENTRAL SCHOOL OF PLANNING AND STATISTICS

02-554 WARSZAWA

Received on 25. 5. 1979

1. CZOCHRALSKA (Warszawa)

\section{METODA PROGRAMO WANIA BILINIO WEGO \\ W NIE WYPUKEYM PROGRAMO WANIU KWADRATOWYM}

STRESZCZENIE

W pracy rozpatruje się problem maksymalizacji wypukłej funkcji kwadratowej na wielościennym zbiorze wypukłym (niekoniecznie ograniczonym).

Problem ten należy do klasy wieloekstremalnych zagadnień programowania matematycznego, a jego maksima lokalne (i w związku z tym również rozwiązanie optymalne) znajdują się w wierzchołkach zbioru rozwiązań dopuszczalnych, co wynika z wypukłości funkcji celu.

Niezależnic od związków z problemem programowania biliniowego sformułowano warunki konieczne $\mathrm{i}$ dostateczne istnienia rozwiązania optymalnego $\mathrm{w}$ rozpatrywanym problemie, których sprawdzenie wymaga jedynie rozwiązania problemów programowania liniowego.

Opierając się na uzyskanych wynikach, opisano algorytm rozwiązujący rozważany problem. 